\title{
Research on Small Satellite Formation Scheme for Earth Observation Task
}

\author{
ZHAO Zheng-yu ${ }^{1,}$ a , CAI Yuan-wen ${ }^{2}$, ZHAO Yi-bin ${ }^{3}$, LI Yan ${ }^{4}$ \\ ${ }^{1}$ Department of Graduate Management, the Equipment Academy, Beijing 101416, China \\ ${ }^{2}$ Department of Space Equipment, the Equipment Academy, Beijing 101416, China \\ ${ }^{3}$ Department of Graduate Management, the Equipment Academy, Beijing 101416, China \\ ${ }^{4}$ Department of Space Equipment, the Equipment Academy, Beijing 101416, China \\ azhaozhengyu1@126.com
}

\section{Keywords: Small Satellite Formation; Earth Observation; Formation Configuration}

\begin{abstract}
The demand of earth observation data is increasing currently and the demand of observation data accuracy, real-time, and continuity is improving continually. Aiming at this problem, the small satellite formation scheme for earth observation task is researched. The significance of the small satellite formation for earth observation task is analyzed. Based on the summary of small satellite formation, the difficulties and enlightenment about small satellite formation for earth observation task have been drawn. Under earth observation task and multi-constrained conditions, the configuration of small satellite formation, the controller of keeping configuration and the scheme of configuration reconstruction are designed.
\end{abstract}

\section{Introduction}

The space-based earth observation system mainly depends on the satellites in orbit. Due to the detecting characteristics of macros copy, dynamic, speediness, accuracy, this system has the unique advantage in solving global environmental problems. And when there are emergency rescue and unexpected disasters, such as earthquake, flood, this system need orbit maneuver or quick launch to achieve the continuous coverage and accuracy evaluation of the local key areas. The time of launching preparation and development cycle of the traditional satellite are too long, so the emergency response ability is weak; the observation ability of the key areas is insufficient; the capacity of continuous observation is inefficient; the function and stability can not meet the actual demand. In contrast, the modern small satellite's quality is light and its size is small; its develop cycle is short and updating of technology is quick; it can be launched quickly with the ability of quick response; it has good maneuverability in order to realize rapid and flexible orbital transfer; its technical performance is advanced and it has high functional density. Modern small satellite provides a new idea for the development of the space-based observation system.

The function which can not be achieved by traditional satellite can be realized through taking use of small satellite formation which makes the multi satellites working together. The demand of observing targets (such as observation accuracy, cover area, revisiting period) of the earth observation task can be realized with rational design of small satellite formation. Research on the application of small satellite formation for earth observation task, is of great significance for the promotion of earth observation task completing ability.

\section{The Analysis of Satellite Formation Research's Present Situation}

Based on the technical advantage, American and other space powers have a relatively mature theory research on satellite formation and have been to space technology validation and application stage.

As the first earth observation satellite of NASA's "New Millennium Program", EarthObserver-1 satellite was launched in 2000 and realized double satellites formation flight with 
Landsat-7[1].GRACE satellite formation flight launched in 2002 is a project of the German Aerospace Center and NASA joint implementation. It uses $\mathrm{K}$ band microwave to measure distance and velocity changes between the two satellites accurately, in order to invert a static and a time variable gravity field with high precision and high spatial resolution[2]. Four "swarn" ESSAIM signal intelligence satellites of France were launched in 2004. Three among them are working satellites, one is a backup satellite. There are 30 kilometers apart from each other. Satellite formation is distributed in rhombus[3]. The German's radar satellite "TanDEM-X" was launched in 2010, with the launched TerraSAR-X forming formation flight closely[4].

Compared with the progress of foreign's satellite formations, domestic progress still remains in theoretical research stage at present. The essence of small satellite formation flight is the relative motion between satellites. In research of applying Hill equation to formation flight relative motion, Zhou Di, He Yan, Ma Yanhong[5] etc did some research in the equation error analysis; in terms of configuration design, Lin Laixing put forward some configurations such as space circle, horizontal circle and the same sub-satellite point, Chen Jihui designed four kinds of common formation configuration with flying around equation[6]. In research of applying relative kinematics equation to formation flight relative motion, under the hypothesis of two bodies condition, Gao Yunfeng, Bao Yinhexi, Li Junfeng derived the exact kinematics equation using master-slave satellite's absolute orbital element; Pan Ligong, Feng Zuren, Liu Jianping etc who considered the effect of the J2 perturbation and elliptical reference described the linear model of the relative motion of formation satellite using the transformation of direct root difference and flat root difference.

Xiao Feng, Xi Xiaoning, Yang Fei, Hao Jigang[7], Liu Jianfeng and so on have carried on the analysis to various perturbation factors. In the aspect of keeping configuration of the satellite formation, Yang Xuerong applied LQR, Wang Zhaokui[8] applied sliding mode variable structure, Ma Kemao designed the Second-order sliding mode control law. In the research of optimization algorithm, various optimization algorithms are more or less applied to the optimal control for satellite formation, such as genetic algorithm, particle swarm algorithm, ant colony algorithm, pseudo-spectral method.

In 2012, China's "Shi Jian 9 Hao" A/B satellites verified the technology of formation flight.

It can be drawed:

(1) There is a big gap in the technology of satellite formation flight between home and abroad. But there is application protect of satellite formation, we need to explore and innovate continually.

(2) The Hill equation is linearized. The analytic solution can be solved when ignoring the perturbation force. Therefore the Hill equation is suitable to analyze the control problem. But in the derivation process, Hill equation is introduced in a number of assumptions and approximations. It also requests that the reference orbit must be a circular orbit. Therefore, the equation error is becoming more and more large accumulating over time.

The accuracy is relatively high in the research of the relative motion of formation satellites through the kinematic method. Theoretically, it is applicable to any situation of the reference orbit eccentricity. And it is not so strict in the relative distance of master-slave satellite as dynamic method. Due to that the current satellite sensors measure relative position and velocity while not orbital element, the application of kinematic method in coordination control of formation flight is limited.

(3) the technology of formation flight is limited by the factors which include perturbation influence, the choice of reference orbit, collision avoidance, energy consumption, responsive time. There is need to combine actual demand of task, plan and select reasonably.

(4) There are many optimization algorithm. But the selected optimization algorithm must be suitable for the demand of satellite formation task. An appropriate has great significance in reducing the amount of calculation and the on-board computer burden, improving work efficiency and the reliability of satellite formation. 


\section{The Difficulties and Enlightenment in Research}

With the further development of the research on small satellite formation, the characteristics and operation mechanism of the small satellite formation were analysed. The difficulties and enlightenment can be drawed in the research:

(1) Stdies found that, some key technologies of satellite formation are still in the space experimental stage, more research still remain in the theoretical research. The applications of satellite formation for earth observation task are much less. So when exploring the applications of small satellite formation for specific earth observation task, more knowledge about earth observation should be mastered. Strengthen the understanding of effective load. After selecting performance indexs, we select the reasonable reference orbit to meet task requirements such as revisiting time, target area coverage performance, spatial resolution and so on.

(2) When designing the small satellite formation, it should be considered that the design of configuration should meet the demand of observation accuracy and coverage performance. At the same time, configuration keeping should also be considered. The limited resources of small satellite would be consumed to keep the configuration. The ability of formation configuration keeping affecting how long the effective formation configuration will last and how much the degree of the task would complete. Small satellite formation performance will influence the whole task cycle length. When designing the scheme overall factors should be grasped. Select some significant performance indexs properly to design the most reasonable scheme.

(3) When the demand of task changes, or small satellites breakdown,or meet emergency demands, the original configuration needs reconstruction. There are many approaches to realize reconstruction. But with the specific formation, the suitable approach need be considered cautiously.

(4) At present most researches focus on the innovations of some key points. In their researches, some specific task was not taken into consideration well.

\section{The Research of Small Satellite Formation Scheme for Earth Observation Task}

1. The selections of small satellite formation's performance index

When the task objectives are different, small satellite formation requirements will be different. The reference satellite's orbit will affect the satellite's revisiting time. In different track environmental satellite formation is under different disturbance factors. In LEO the influence of J2 perturbation is lager than the atmospheric drag perturbation. While in the high track if $\mathrm{J} 2$ perturbation, lunisolar gravitational perturbation, solar radiation pressure perturbation should be considered or not depend on the observation accuracy and track altitude. Combined with different satellite formation, the energy consumed in keeping the formation will be different. In order to maintain the overall performance of the formation, the overall energy consumption of the formation and the balance of energy consumption of each satellite should be considered together. In emergency task, the performance of response time should be outstood. When the requirement of response time performance is not so strict, the overall and balance energy consumption should be outstood. Different formations have different observation precision and target coverage performance. So when the satellite formation takes the earth observation task, the performance indicators should be selected reasonably to ensure the formation optimal, according to the specific task requirements.

2. The design of small satellite formation configuration

First of all, analyse the specific earth observation task. According to the task requirements, confirm the indexes which can evaluate the completion ability of task. Then analyse the application characteristics of different tracks for earth observation task. The satellite formation in the low earth track has relatively high resolution. The satellite formation in the high track has relatively lager coverage area, but its resolution is relatively low. In the low track atmospheric drag perturbation'influence is lager than lunisolar gravitational perturbation and solar radiation pressure perturbation, while in the high track its influence will decrease. 
Next step is to research the relative motion dynamics equation and kinematical equation. Find out their same characteristics and their different preponderances. But the dynamics equation is easy to get analytic solution. Kinematic equation has less limitiations than Dynamics equation. And kinematic equation has some innovative developments. Research the basic formation configuration which had been designed. We can select basic formation configuration for the task or design a new formation configuration. The new formation configuration can be a innovative one which is based on the basic ones, or the combination of some basic ones, or a completive one different from the ones had been designed. Finally, through the simulation analysis, verify the validity of the formation configuration and the improvement of the performance.

When the reference track is circle, relative motion dynamics equations are as follows:

$$
\begin{aligned}
& x-2 n y-3 n^{2} x=f_{x} \\
& y+2 n x=f_{y} \\
& z+n^{2} z=f_{z}
\end{aligned}
$$

In the formula, $f_{x}, f_{y}, f_{z}$ were components of the three axes of the orbital coordinate system.

When the reference track is not circle, the orbital angular velocity is not constant, the relative motion equations are as follows:

$$
\begin{aligned}
& x-2 n y-n^{2}\left(2 \frac{r}{p}+1\right) x-n y=f_{x} \\
& y+2 n x+n^{2}\left(\frac{r}{p-1}\right)+n x=f_{y} \\
& z+n^{2} \frac{r}{p} z=f_{z}
\end{aligned}
$$

In the formulas, $n$ is the rate of change of the orbital angular velocity, $r$ is the distance from the earth to the reference satellite, $p$ is semi-parameter of the reference orbit, it can be calculated using the follow equation:

$$
p=a\left(1-e^{2}\right)
$$

In the formula, $a$ is the reference orbit semi-major axis, $e$ is eccentricity ratio.

The relative motion equations of kinematics are as follows:

In the formulas,

$$
\begin{aligned}
& x=-a e_{A} \cos (n t+\theta) \\
& y=2 a e_{A} \sin (n t+\theta)+a \Delta \lambda \\
& z=a \Delta i \sin (n t+\psi)
\end{aligned}
$$

$$
\begin{aligned}
& e_{A}=\sqrt{\left(e_{s} \cos \phi-e_{m}\right)^{2}+\left(e_{s} \sin \phi\right)^{2}} \\
& \theta=\arctan \left(\frac{e_{s} \sin \phi}{e_{s} \cos \phi-e_{m}}\right) \\
& \phi=\Delta \lambda-\left(\omega_{s}-\omega_{m}\right)+(k-\varphi) \\
& \psi=\omega_{m}-\varphi \\
& \Delta \lambda=\left(\omega_{s}-\omega_{m}\right)+\left(M_{s}-M_{m}\right)-(k-\varphi)
\end{aligned}
$$

The "s", "m" in the subscript respectively represent the surrounding satellite and the reference satellite, $\omega$ is argument of perigee, $\mathrm{M}$ is mean anomaly, $\mathrm{k}$ is the geocentric angle from the ascending node of the surrounding satellite to the relative ascending node of two satellites, $\varphi$ is the geocentric 
angle from the ascending node of the reference satellite to the relative ascending node of two satellites.

3. The control of keeping small satellite formation configuration

Because of the model error and distraction from the space, the formation configuration will gradually deviate from the designed configuration. Then we need to keep the formation configuration.

If the request is not very high, we can use the control technique of traditional satellite or constellations for reference. If the request is strict, it is necessary to design the controller to keep the formation configuration. Formation keeping control focuses on the underlying control, namely the control method.

Autonomous coordination control is the characteristic different from the control method for other types of satellite. Analysing the main factors results in the configuration's deviating. According to them, establish a rational model. A major advantage of satellite formation'controlling is to use the relative navigation information between two satellites. And it puts the nacigation system for measuring the relative state and the control system to unify. Most early researches put the linearized dynamics equation as a dynamic model of relative control. And design the controller of formation keeping with linear control method. In order to improve the precision and reduce the linearization error, the nonlinear configuration control methods can be used.

In order to guarantee the operational use time, the hold time of the effective configuration should be increased by optimizing the algorithm. When carrying on the detailed design, innovative applications can be researched from the two aspects of the model and algorithm. Finally, prove the designed configuration keeping control is scientific and effective through the simulation analysis.

4. The control of small satellite formation reconfiguration

The configuration refactoring of small satellite formation shows the outstanding features of functional diversity and flexible operational mode. There is a key point that the configuration refactoring requires satellites's synergic movement to complete the orbital transfer.

After the formation deployed in space, due to the mission or technical requirements change, the established formation need to be restructured. The resolution of the synthetic aperture system can be adjusted and the satellites's fuel can be saved by adjusting the configuration size to change the formation baseline. The distributed satellites' collaborative work direction can be changed by adjusting formation's flying plane. When one satellite of the formation breakdown, the satellite's phase of the formation can be adjusted to degrade system and avoid collision.

Select the target configuration for the practical application background. Use the maneuver method of single satellite or constellation for reference. Select pulse or continuous thrust based on the hardware condition or task requirement. Plan the path and optimize it. Take collosion avoidance into consideration. Select the optimal algorithm. There are some inevitable problems in algorithms which had been researched by many scholars. There is a problem that the chattering can be eliminated in the algorithm of sliding mode variable structure. The linear model has interference error. We can use only one algorithm and improve it, or mix some algorithms together. Finally verify the validity by simulation.

5. The design of overall research project

First, design the formation configuration for the specific earth observation task. Then design the controller of keeping the formation configuration. Design the configuration restruction. After these designs completed, do the simulation to verify the validity. The concrete implementation plan is shown in the figure below: 


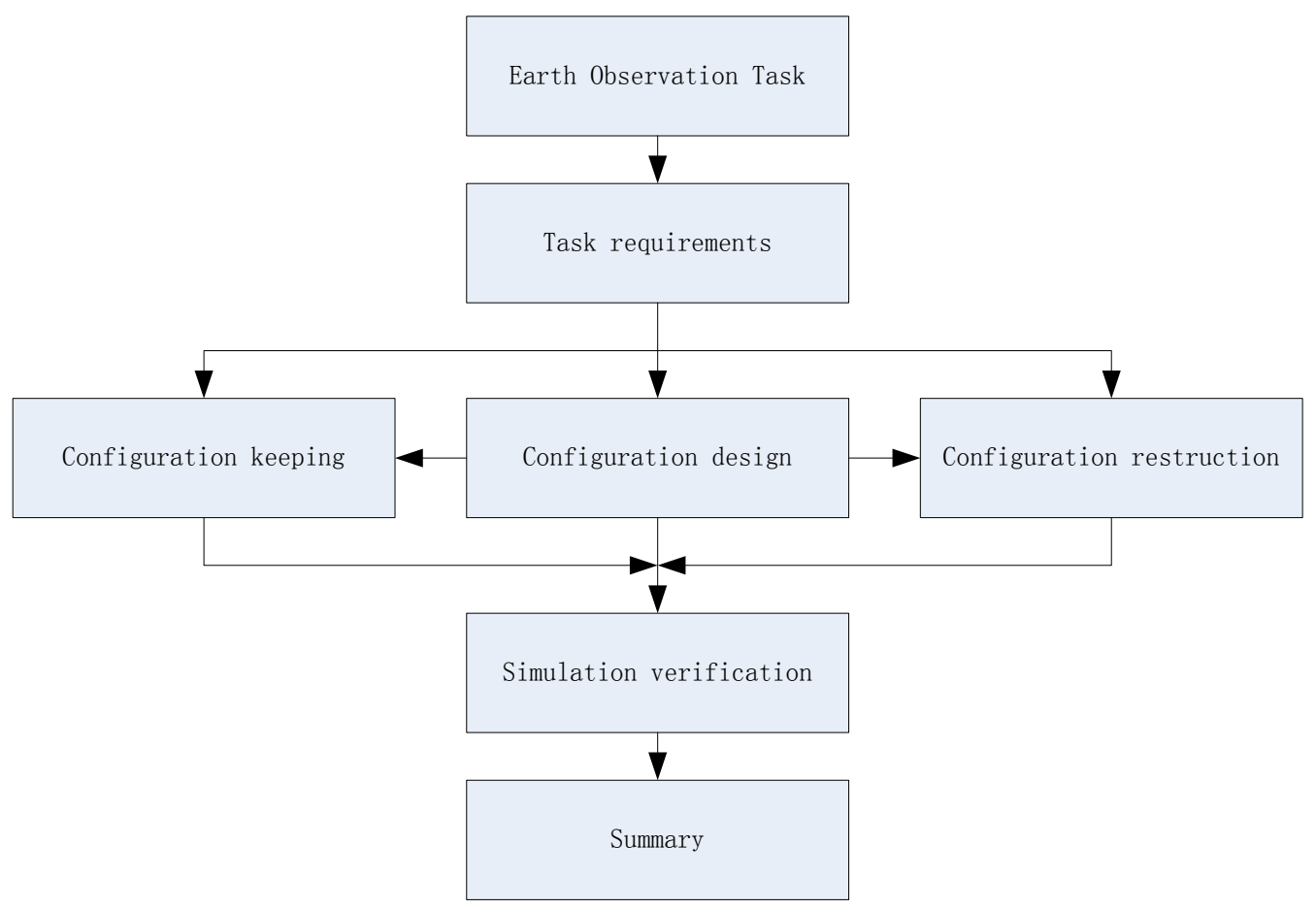

Fig.1 The overall plan

\section{Summary}

Based on the summary of research status of the small satellite formation, the difficulties and enlightenments of designing the small satellite formation for earth observation can be obtained. There is a far-reaching significance for small satellite formation in the future earth observation application.

\section{References}

[1] Liu Ming. The research of relative motion and formation control of the small satellite formation[D]. Shang hai jiao tong university, 2007.

[2] Han S C, Jekeli C, Shum C K. Static and temporal gravity field recovery using grace potential difference observables[J]. Advances in Geosciences, 2003.

[3] Chang Xin, Xiang Sheng-yu. The analysis of the French space force[J]. Computer knowledge and Technology, 2009.

[4] Li He, Qin Zhi-yuan, Chou Gong-run, Ji Yuan. The research progress of TerraSAR-X and TanDEM-X project[J]. The first national remote sensing remote control technology seminar, 2008.

[5] Ma Yan-hong, The Error Analysis of C-W Guidance[J]. Aerospace Control, 2009.

[6] Chen Ji-hui, Xiong Zhi, Wang Rong, etc. The Configuration Kinematics Design of Small Satellies Flying-around formation[J]. Aerospace Control, 2009.

[7] Hao Ji-gang. Study on Formation Configuration Control of Distributed Satellites[D]. Hunan Changsha: National university of defense technology, 2006.

[8] Wang Zhao-kui, Zhang Yu-lin. Variable Structure Control for Precision Formation-keeping of Distributed Satellites[J]. Aerospace Control, 2005. 